\title{
Interactions Between the Circadian Clock and Heme Oxygenase in the Retina of Drosophila melanogaster
}

\author{
Milena Damulewicz ${ }^{1}$ - Agnieszka Loboda ${ }^{2}$ - Alicja Jozkowicz ${ }^{2}$. Jozef Dulak ${ }^{2}$ • \\ Elzbieta Pyza ${ }^{1}$
}

Received: 25 February 2016 / Accepted: 1 August 2016 / Published online: 13 August 2016

(C) The Author(s) 2016. This article is published with open access at Springerlink.com

\begin{abstract}
The Drosophila retina has an autonomous peripheral circadian clock in which the expression of the gene encoding heme oxygenase (HO) is under circadian control with the ho mRNA peaking at the beginning of the day and in the middle of the night. The function of $\mathrm{HO}$ in the retina is unknown, but we observed that it regulates the circadian clock and protects photoreceptors against DNA damage. The decline in $\mathrm{HO}$ level increases and decreases the expression of the canonical clock genes period (per) and Clock $(\mathrm{Clk})$, respectively. The opposite result was observed after increasing $\mathrm{HO}$ expression. Among three products of $\mathrm{HO}$ activity-carbon monoxide (CO), ferrous ions, and biliverdin - the latter has no effect on per and $C l k$ expressions, but $\mathrm{CO}$ exerts the same effect as the increase of $h o$ expression. This suggests that $\mathrm{HO}$ action on the clock is mediated by $\mathrm{CO}$, which may affect $C l k$ expression during the day and the level of per expression. While ho expression is not stimulated by nitric oxide (NO), NO has the same effect on the clock as HO, increasing Clk expression and decreasing the expression of per.
\end{abstract}

Keywords Drosophila $\cdot$ Circadian clock $\cdot$ Heme oxygenase · Retina $\cdot$ Carbon monoxide $\cdot$ Nitric oxide

Electronic supplementary material The online version of this article (doi:10.1007/s12035-016-0026-9) contains supplementary material, which is available to authorized users.

Elzbieta Pyza

elzbieta.pyza@uj.edu.pl

1 Department of Cell Biology and Imaging, Faculty of Biology and Earth Sciences, Jagiellonian University, 30-387 Krakow, Poland

2 Department of Medical Biotechnology, Faculty of Biochemistry, Biophysics and Biotechnology, Jagiellonian University, 30-387 Krakow, Poland

\section{Introduction}

Heme oxygenase (HO) is an enzyme that degrades heme to carbon monoxide (CO), ferrous ions, and biliverdin. In mammals, there are two isoforms of $\mathrm{HO}$ encoded by two different genes, inducible HO-1 and constitutive HO-2. Although they differ in distribution and functions, they both act as cytoprotective and anti-apoptotic agents in an organism, by scavenging reactive oxygen species (ROS; reviewed in [1]). In Drosophila melanogaster, there is only one gene encoding $\mathrm{HO}$ [2] that plays an important role in development [3] and in controlling the signaling pathway of DNA damage [4]. Any other functions of $\mathrm{HO}$ in insects are still unknown.

In the suprachiasmatic nuclei ( $\mathrm{SCN})$, the site of the mammalian circadian pacemaker for behavioral rhythms, $\mathrm{HO}$ activity changes during the day, reaching the maximum during the night. This pattern is maintained in constant darkness, suggesting that the activity of $\mathrm{HO}$ is controlled by the circadian clock [5]. Moreover, changes in the heme level influence the expression of clock genes [6,7] as well as the degradation of the clock protein PERIOD 2 (PER2) [7]. This specific function of heme is tissue-dependent [8]. In D. melanogaster, cyclic expression of the ho gene has been found in whole head homogenates [9], but nothing is known about the role of $\mathrm{HO}$ in the circadian system.

In Drosophila, as in all other animals, apart from the central pacemaker, there are peripheral clocks, one of the most studied being located in the retina. This oscillator generates cyclic processes in the retina and partly in the lamina, the first neuropil of the optic lobe [10-12]. The molecular mechanism of the retina clock is similar to that of the pacemaker and is based on several interlocked feedback loops $[10,13]$. In the main negative feedback loop, PERIOD (PER) and TIMLESS (TIM) proteins inhibit transcription of their own genes per and tim, the core clock genes. The expression of per and tim is 
regulated by the transcription factors CLOCK (CLK) and CYCLE (CYC) that, late in the evening, dimerize and enter the nucleus, binding to the regulatory E-box sequences in the promoters of per and tim, inducing their expression. At the same time, CLK and CYC control the expression of clockcontrolled genes, and one of them could conceivably be $h o$. In turn, PER and TIM proteins dimerize late at night and are transported to the nucleus, where they bind to CLK/CYC and inhibit their activity, thereby halting the transcription of per and tim. In contrast to the pacemaker, CRYPTOCHROME, a circadian photoreceptor, acts as a repressor in the retinal clock and inhibits CLK activity [14].

Since HO may play a cytoprotective function and the retina is a site of intense physiological processes of phototransduction that generates high levels of ROS [15], we examined whether this protein is cyclically expressed in the retina, in parallel to circadian rhythms in the fly's visual system [16], and whether HO influences the retinal clock and protects photoreceptors against degeneration. We found that ho gene expression is controlled by the circadian clock and $\mathrm{HO}$ also regulates the clock of $D$. melanogaster. Moreover, HO reduces DNA damage after exposure to UV and white light.

\section{Materials and Methods}

\section{Animals}

We used the following strains of D. melanogaster: wild-type Canton S, Clk ${ }^{J^{r k}}$ (mutant in which premature stop codon disrupts the activation domain of the CLOCK protein) [17]; $c y c^{0}$ (recessive null mutant of the cycle gen) [18]; per $^{01}$ (null mutant of the period gene) [19]; Rh1-Gal4 (strain which expresses GAL4 under the control of the rhodopsin1 promoter) [20]; repo-GAL4 (strain which expresses GAL4 under the control of the repo (reversed polarity) promoter-glial cell marker); UAS- $g f p$, UAS-hoRNAi (strain which expresses dsRNA for ho gene under the control of UAS sequence) [3]; and UAS-Valium10-gfp (control for RNAi flies) [21]. Flies were maintained under $12 \mathrm{~h}$ of light and $12 \mathrm{~h}$ of darkness (LD12:12) conditions or in constant darkness (DD) and at a constant temperature of $24{ }^{\circ} \mathrm{C}$.

\section{In Vitro Experiments}

For in vitro experiments, we used Schneider 2 cells (S2; Invitrogen) derived from a primary culture of late-stage (2024 h old) D. melanogaster embryos [22]. Cells were cultured in Schneider's medium (Invitrogen) with $10 \%$ fetal bovine serum (Invitrogen) and $100 \mathrm{U} / \mathrm{ml}$ penicillin $/ 100 \mu \mathrm{g} / \mathrm{ml}$ streptomycin (MP Biomedicals) at $25{ }^{\circ} \mathrm{C}$.

\section{Experimental Procedure}

To detect cyclic expression of $h o$ in the retina, we analyzed ho messenger RNA (mRNA) level at five time points in LD12:12 and DD conditions: $1 \mathrm{~h}$ after the beginning of the day (Zeitgeber Time, ZT1)/the subjective day (Circadian Time, CT1); $4 \mathrm{~h}$ after the beginning of the day (ZT4)/the subjective day (CT4); $1 \mathrm{~h}$ after the beginning of the night (ZT13)/the subjective night (CT13); $4 \mathrm{~h}$ after the beginning of the night (ZT16)/the subjective night (CT16); and $8 \mathrm{~h}$ after the beginning of the night (ZT20)/the subjective night (CT20). ZT0 indicates the beginning of the light/day phase and ZT12 stands for the beginning of the night/dark phase in LD12:12, whereas CT0 and CT12 stand for the beginning of the subjective day and the beginning of the subjective night, respectively, in DD. It has been reported that significant differences in the expression level of many clock-controlled genes occur at these time points $[10,12]$. In addition, we examined $h o$ expression profile exclusively in glial cells which were sorted out of the brain according to the methods published previously [10]. For this experiment, we used repo $>g f p$ and repo $>h o R N A i, g f p$ strains and examined ho mRNA level at ZT1, ZT4, ZT13, and ZT16.

To study the effects of $\mathrm{HO}$ on the clock in the retina, we applied several chemicals and examined the expression of clock genes per and $C l k$. Flies were starved for $12 \mathrm{~h}$ with water available ad libitum; next, they were fed for $12 \mathrm{~h}$ (until decapitation) with $6 \%$ glucose solution supplemented with: $100 \mu \mathrm{M}$ of hemin chloride (Calbiochem), a heme oxygenase activator; $100 \mu \mathrm{M}$ of tin protoporphyrin IX (SnPPIX, Frontier Scientific), a heme oxygenase inhibitor; $500 \mu \mathrm{M}$ of carbon monoxide-releasing molecule 2 (CORM-2, Sigma-Aldrich), a carbon monoxide (CO) donor; $500 \mu \mathrm{M}$ of $S$-nitroso- $N$ acetylpenicillamine (SNAP, Sigma-Aldrich), a nitric oxide (NO) donor; or $500 \mu \mathrm{M}$ of biliverdin dihydrochloride (BV, MP Biomedicals). Control flies were fed with $6 \%$ glucose solution with addition of $\mathrm{NaOH}$ (solvent for hemin, SnPPIX, SNAP, and BV). In the case of the experiment with CORM-2, control flies were fed with $500 \mu \mathrm{M}$ of the inactive compound (iCORM) which does not release CO (prepared in DMSO, similarly to CORM-2, but left overnight in room temperature to liberate $\mathrm{CO}$ ).

To establish the proper concentration of SNAP for experiments, we examined the ROS level in the brain of Drosophila after SNAP application. It has been found that the application of SNAP specifically increases ROS production in cells [23]. First, flies were starved for $12 \mathrm{~h}$ and then fed with 100,250 , or $500 \mu \mathrm{M}$ of SNAP for $12 \mathrm{~h}$. Then, brains were isolated and treated with trypsin/EDTA for $1 \mathrm{~h}$ at $37^{\circ} \mathrm{C}$. The obtained cells were centrifuged for $10 \mathrm{~min}$ at $10,000 \times g$ and then resuspended and incubated in phosphate-buffered saline PBS with $10 \mu \mathrm{M}$ ROS Detection Reagents (Invitrogen) for $1 \mathrm{~h}$ at $37{ }^{\circ} \mathrm{C}$. Next, they were centrifuged again, resuspended in PBS, and analyzed by flow cytometry. The cells obtained 
from flies not fed with SNAP served as a control. As a negative control, cells without ROS dye treatment were used.

To determine DNA damage in the retina photoreceptors after light exposure and different $\mathrm{HO}$ levels in cells, flies were kept in DD for 5 days and starved for $6 \mathrm{~h}$; water was available ad libitum. Next, they were fed for $6 \mathrm{~h}$ (until decapitation) with $6 \%$ glucose in water supplemented with $\mathrm{HO}$ activator, $100 \mu \mathrm{M}$ hemin chloride (Calbiochem), or HO inhibitor, $100 \mu \mathrm{M}$ tin protoporphyrin IX (SnPPIX, Frontier Scientific). The control group was fed only with glucose. All groups of flies were exposed to $1 \mathrm{~h}$ UV light pulse (100 lx) at CT0 and then to $3 \mathrm{~h}$ white light (1500 lx) emitted from UV and white light UVECX26 and TFX53WL transluminators (Vilbert Lourmat), respectively. After that they were decapitated and fixed in $4 \%$ paraformaldehyde. Cryosections were prepared and immunodetection with anti-8-hydroxyguanosine primary antibody (1:500, overnight), which labels oxidative DNA damages, was carried out. On the following day of the procedure, HRP/DAB (ABC) detection kit was used according to the manufacturer's protocol (Abcam). Negative control was prepared without the primary antibody. In positive control, heads were decapitated and incubated for $4 \mathrm{~h}$ in Schneider's medium supplemented with etoposide, a cytotoxic compound. We also used two different control groups: the first group was fed with glucose and exposed to UV and white light and the second one was collected in DD.

Clock gene expression was also studied in S2 cells which were cultured in Schneider's medium supplemented with $100 \mu \mathrm{M}$ of hemin, SnPPIX, CORM-2, BV, or SNAP for $6 \mathrm{~h}$. Time of exposure and the concentration of the chemicals listed above were established during our preliminary study. Control cells were treated with the solvent or with iCORM. S2 cells do not have circadian clocks, but express some clock and clockcontrolled genes and can be used to study certain aspects of the circadian clock functioning [24].

\section{RNA Isolation and $q P C R$}

Males, 7 days old, were decapitated at ZT1, ZT4, ZT13, and ZT16 under LD12:12 or at CT1, CT4, CT13, and CT16 under DD. Heads were fixed in $100 \%$ ethanol for $2 \mathrm{~h}$ and retinas were isolated. Approximately 30 flies were used for each time point, and each experiment was repeated at least three times.

Total RNA isolation was performed using TriReagent (MRC Inc.) according to the manufacturer's protocol. The complementary DNA (cDNA) for polymerase chain reaction (PCR) amplification was prepared from $1 \mu \mathrm{g}$ total RNA using Superscript II reverse transcriptase (Life Technologies) according to the provider's instruction. Gene expression was examined using TaqMan Gene Expression Assays labeled with 6'-FAM (Applied Biosystems) and 7500 Fast RealTime PCR System (Applied Biosystems). The following genes were examined: Ribosomal protein 32 (rpl32,
Dm02151827_g1) as a reference gene; period (per, Dm01843683_g1); and Clock (Clk, Dm01795381_g1). cDNA, diluted 1:10, was used for quantitative PCR. Each experiment was repeated at least three times. The expression of ho gene was examined using SYBR Green Master Mix (Applied Biosystems) and the following primers: forward primer: 5'-ACCATTTGCCCGCCGGGATG; reverse primer: 5'-AGTGCGACGGCCAGCTTCCT; for rpl32: forward primer: 5'-AGAAGCGCAAGGAGATTGTC; reverse primer: 5'-ATGGTGCTGCTATCCCAATC. Product specificity was assessed by melting curve analysis, and selected samples were run on $1 \%$ agarose gels for size assessment.

Data were collected as raw $C_{\mathrm{T}}$ values and analyzed using the $2^{-\Delta \Delta C \mathrm{~T}}$ method. Gene expression was normalized on an arbitrary scale with control (or ZT1) as 1.0.

\section{Statistical Analysis}

Statistical analysis was performed using non-parametric analysis of variance (ANOVA) Kruskal-Wallis test and then Tukey's test or non-parametric Mann-Whitney test to compare differences between groups fixed at different time points or between experimental and control groups. Statistica 7.0 software was used for analysis, and statistically significant differences were at $p<0.05$.

\section{Results}

\section{Expression of ho in LD12:12 and DD}

The examination of ho mRNA level at five time points during the day in the retina showed that ho expression changes during the day and night in LD12:12 (Fig. 1a), with two peaks at ZT1 and ZT16. The differences between ZT1, ZT16, and other time points were statistically significant. This bimodal pattern was maintained in constant darkness (DD; Fig. 1b) and the rhythm was abolished in per $^{O}$ (Fig. 1c), indicating that ho expression is controlled by a circadian clock. The examination of ho expression pattern in glial cells showed that ho transcript levels change during the day and night in LD12:12, with a peak at ZT16, and higher levels of mRNA at ZT4 than at ZT13 (Fig. 1d). It means that ho expression is also cyclical in glial cells, but has a different daily pattern than in the retina.

\section{Effects of HO, CO, and NO on the Retina Clock}

To verify a possible effect of $\mathrm{HO}$ on the clock, we fed flies with an activator (hemin) or inhibitor (SnPPIX) of heme oxygenase activity. In our preliminary experiments, we have found that $100 \mu \mathrm{M}$ hemin increases ho gene expression (Fig. S1), and we used this concentration for further experiments. $\mathrm{HO}$ activation by hemin was accompanied by a decrease in per expression in the retina of 
a ho expression

Canton S LD 12:12
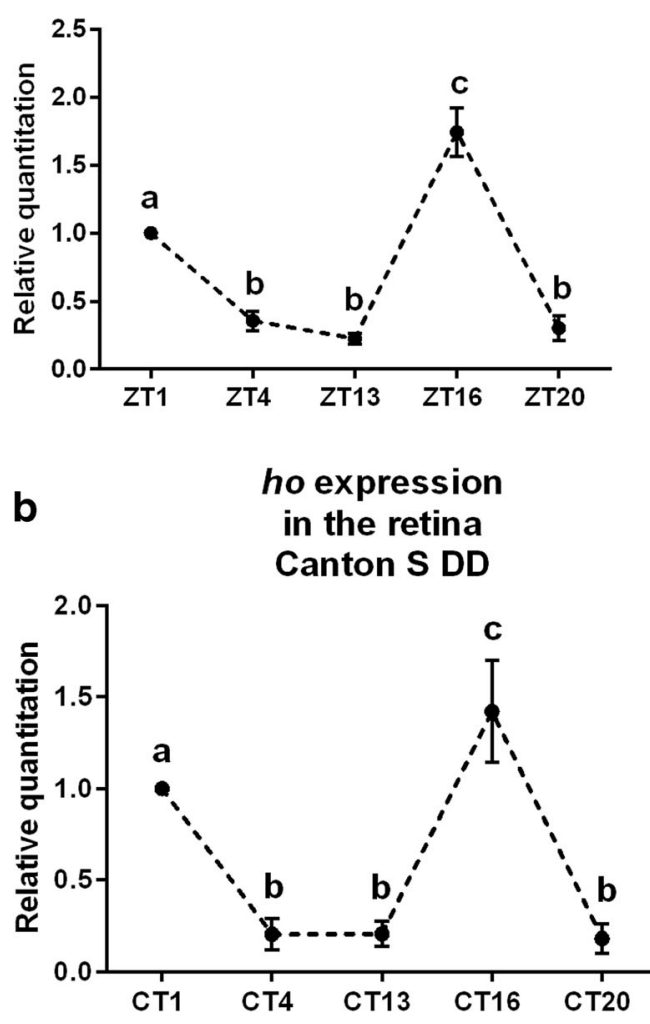

ho expression

C

in the retina

c per ${ }^{01}$ mutant

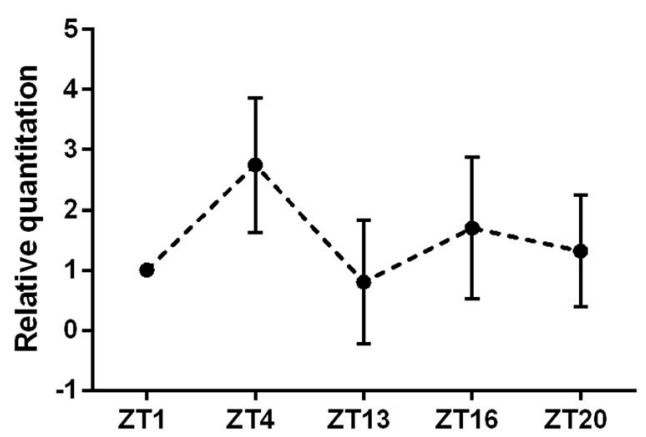

d

ho expression

in glial cells

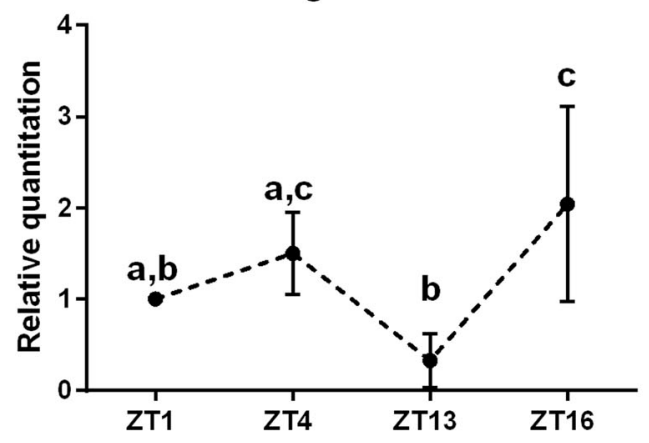

Fig. 1 Expression of ho gene in the retina of wild-type D. melanogaster at four time points in LD12:12 (a) and DD conditions (b) and in per ${ }^{\text {OI }}$ mutant (c) in LD12:12 as well as in glial cells (d). Statistically significant differences between time points are marked with different letters. Graph presents relative quantitation of steady-state $\mathrm{mRNA}$ level $\pm \mathrm{SD}$; data are normalized to ZT1 in LD12:12 or CT1 in DD (value $=1.0$ ). Non-parametric Kruskal-Wallis test, parametric ANOVA Tukey's test: $p<0.05$

flies in comparison to the control flies fed with glucose (Fig. 2a), but the rhythm of per mRNA was maintained, with a peak atZT16 (Fig. S2A). Surprisingly, at ZT1, per mRNA level was significantly higher than that in the control, while at other time points
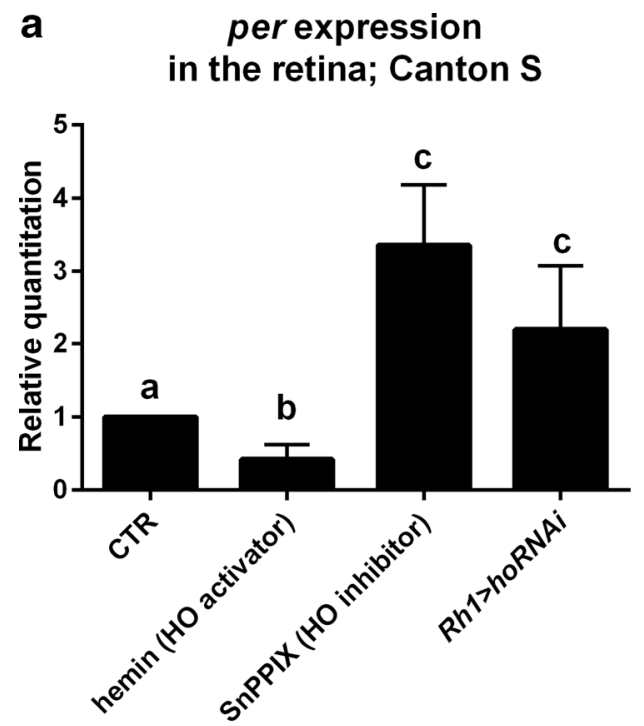

b Clk expression in the retina; Canton S

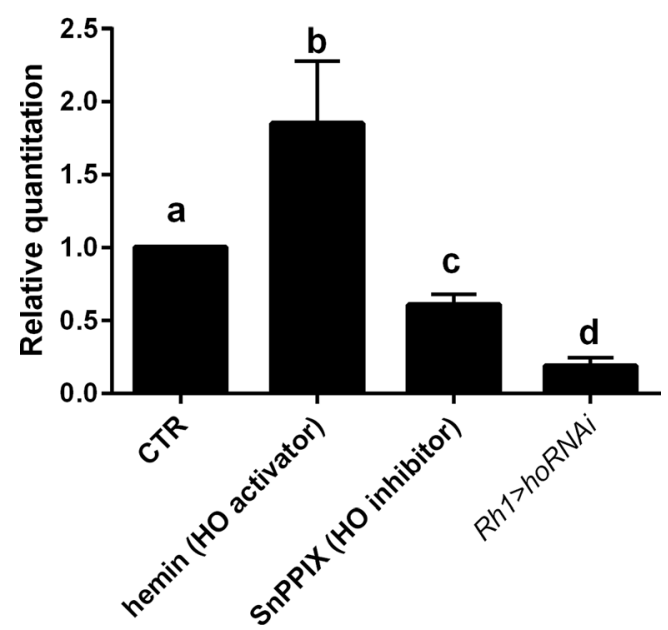

Fig. 2 Effects of hemin $(100 \mu \mathrm{M})$ and SnPPIX $(100 \mu \mathrm{M})$ treatments as well as ho silencing in the photoreceptors ( $R h l>h o R N A i$ flies) compared with the control (glucose with solvent for chemical treatment or Rhl $>$ Valium 10 flies, respectively) on per (a) and Clk (b) gene expressions in the retina. Statistically significant differences are marked with different letters. Non-parametric Kruskal-Wallis test and parametric ANOVA Tukey's test were used, $p<0.05$; data are normalized to control $(\mathrm{CTR}=1)$. Bars and graphs represent changes in mRNA levels (average of all time points $\pm \mathrm{SD}$ ) 
lower (Fig. S2B). In contrast, $C l k$ expression was increased after hemin treatment, except at ZT4 (Fig. 2b). Moreover, in flies treated with hemin, the $C l k$ mRNA oscillations were flat, and a statistically significant difference in mRNA level was detected only between ZT4 and ZT16 (Fig. S2C, D).

The effects of SnPPIX (HO inhibitor) on per and Clk expressions were opposite to those of hemin. per mRNA level was similar during the day, except for a trough at ZT4 (Fig. S2E). Flies fed with SnPPIX had higher per expression, except at ZT4 (Fig. 2a and Fig. S2F). In contrast, Clk mRNA level was lower after SnPPIX treatment than that in the control (Fig. 2b), except at ZT1. Moreover, the expression of $C l k$ in experimental flies was the highest at the beginning of the day (ZT1), earlier than in control flies (Fig. S2G, H).

In addition to the chemical modulation of $\mathrm{HO}$ activity by its activator or inhibitor, we used RNAi technology in transgenic flies to decrease ho expression specifically in the retina. Rhl-GAL4 females were crossed to UAS-hoRNAi males to decrease $\mathrm{HO}$ level in photoreceptors R1-R6 which carry rhodopsin Rh1 in their offspring. As a control, we used Rh1-GAL4 crossed to UAS-Valium 10 flies. In the retina of experimental flies, ho expression was lower than that in control flies (Fig. S3A). After silencing of ho, the expression of per was higher than that in control flies at all time points studied (Fig. 2a and Fig. S3B), and the daily pattern of per expression was the same as that in the control (Fig. S3C). In turn, Clk expression was strongly decreased in the experimental flies when compared with the control (Fig. 2b and Fig. S3D), but its daily pattern was not changed (Fig. S3E).

The silencing of ho in glial cells abolished the daily rhythm of per and Clk expressions, indicating that the clock was disrupted since in the control both mRNAs cycle during the day, with peaks at ZT16 (per) and ZT4 (Clk; Fig. 3a, b). After ho silencing in glial cells, per and $C l k$ mRNA levels in those cells were the same at four time points studied (data not shown). Because the effect of ho silencing is different in glial cells than in the retina described above, it suggests that glial clock is different from that of the retina photoreceptors.

To assess whether HO modulates the clock mechanism directly or has a rather indirect effect, we treated flies with $\mathrm{HO}$ activity products: $\mathrm{CO}$, using $\mathrm{CO}$ donor CORM-2, or BV. Flies fed with $500 \mu \mathrm{M}$ of CORM-2 as well as $500 \mu \mathrm{M}$ or $1 \mathrm{mM}$ of BV did not show any changes in per and Clk expressions (Fig. 4a, b and Fig. S4A, B), while a higher CORM-2 concentration (1 mM) was lethal for flies. We also examined the effect of $\mathrm{NO}$ donor because of a possible cross-talk between $\mathrm{NO}$ and $\mathrm{HO}[25,26]$. In our preliminary study, we found that the effective concentration of SNAP, established by measuring the ROS level, was $500 \mu \mathrm{M}$ (Fig. S5). The application of SNAP decreased per and
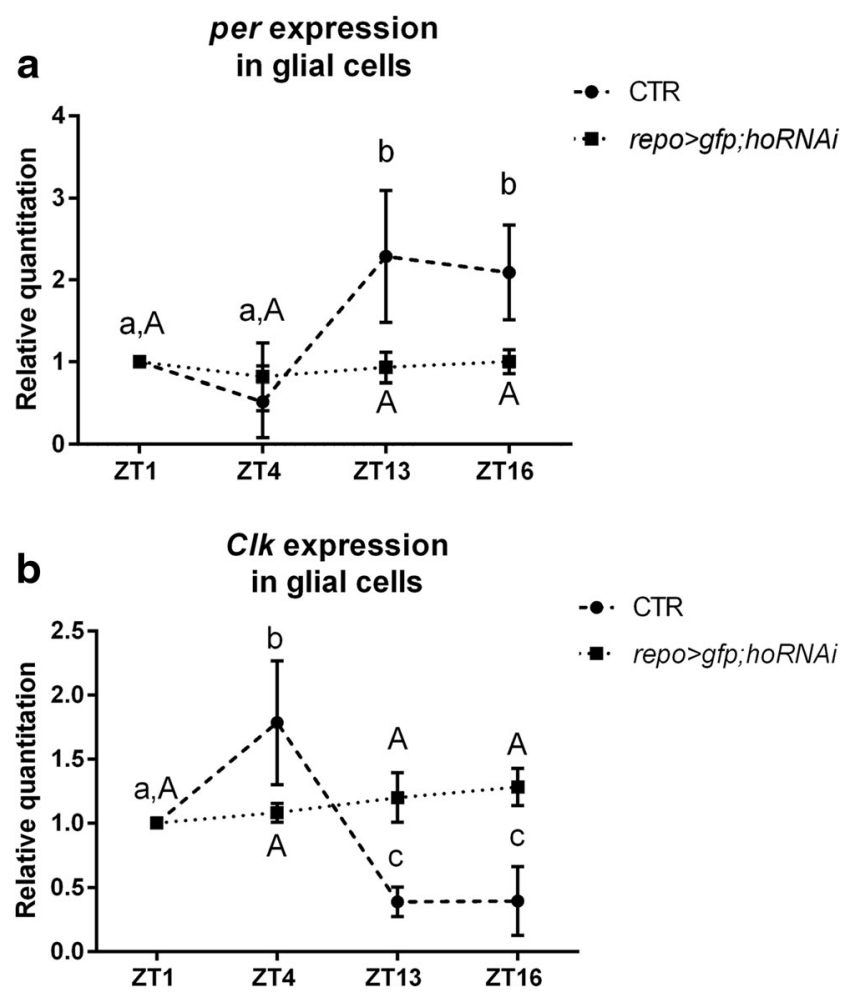

Fig. 3 Effect of ho silencing in the glial cells on per (a) and Clk (b) expressions in the glial cells of repo $>$ hoRNAi;gfp Drosophila strain. As a control, repo $>g f p$ strain was used $(C T R)$. Statistically significant differences are marked with letters (lowercase letters for control and uppercase letters for hoRNAi); data are normalized to ZT1 as value 1 . Non-parametric Kruskal-Wallis test, parametric ANOVA Tukey's test: $p<0.05$. Graph represents average clock gene expression level \pm SD

increased $C l k$ expressions in comparison with the control treated with a solvent (Fig. 4a, b). This effect was observed at each time point studied and was similar to that obtained after feeding flies with hemin (Fig. S6A, B).

\section{Effects of HO and NO on Clock Gene Expression in the Retina of $\mathrm{Clk}^{\mathrm{Jrk}}$ Mutant Flies}

In mammals, $\mathrm{NO}$ and $\mathrm{CO}$ bind to clock proteins acting as transcription factors and change their ability for DNA binding [27]. Looking for a similar mechanism in Drosophila, we compared per and Clk expressions in wild-type flies, $C l k^{J r k}$ and $c y c^{0}$ mutants after feeding with hemin, SnPPIX, or SNAP. Clk ${ }^{J r k}$ flies fed with hemin or SNAP did not show differences in per expression, but surprisingly, per mRNA level was significantly decreased after SnPPIX treatment (Fig. 5a). On the other hand, $c y c^{0}$ mutant fed with hemin showed a decrease in per expression, whereas SnPPIX exerted an opposite effect on per expression (Fig. 5b). $c y c^{0}$ flies after SNAP treatment did not show differences in per mRNA level compared with the control (Fig. 5b). In contrast, Clk expression in $c y c^{0}$ mutants fed with hemin or SnPPIX 


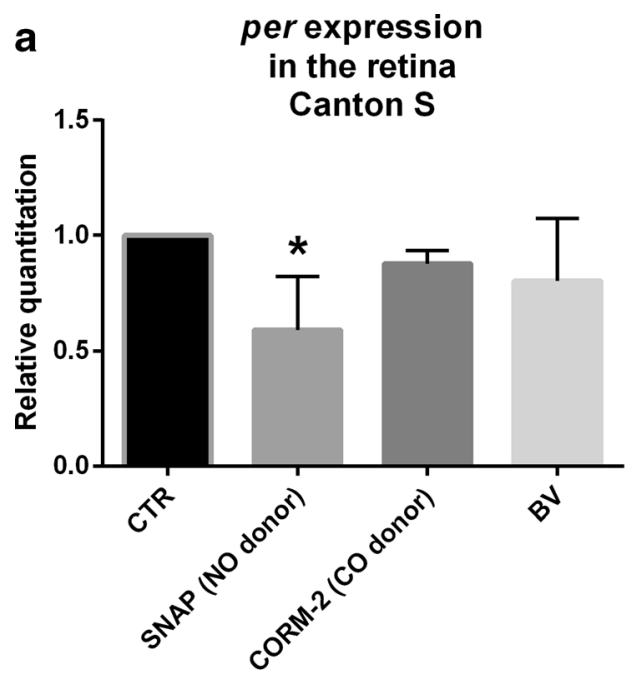

b

Clk expression in the retina; Canton S

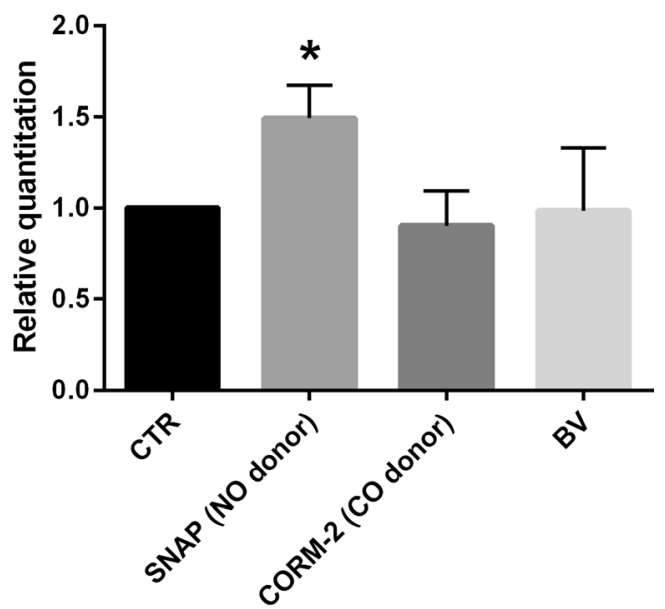

Fig. 4 Effects of SNAP $(500 \mu \mathrm{M})$, CORM-2 $(500 \mu \mathrm{M})$, and biliverdin $(B V ; 500 \mu \mathrm{M})$ treatments compared with control on per (a) and Clk (b) gene expressions in the retina of wild-type $D$. melanogaster. Data are normalized to the control as value 1 . Statistically significant differences are marked with stars, $p<0.05$. Mann-Whitney test: $p<0.05$. Bars represent average clock gene expression $\pm \mathrm{SD}$

was similar to that in control flies, but flies fed with SNAP had lower Clk mRNA level (Fig. 5c). These results indicate that changes in $\mathrm{HO}$ activity and an increase in NO level affect the clock through CLOCK protein. On the other hand, the observed changes in per expression after SnPPIX suggest another, not clock-dependent function of PER that has already been reported in the visual system of Drosophila [10,12].

Fig. 5 Effects of hemin $(100 \mu \mathrm{M})$, SnPPIX $(100 \mu \mathrm{M})$, and SNAP $(500 \mu \mathrm{M})$ treatments compared with control on per $(\mathbf{a}, \mathbf{b})$ and $C l k(\mathbf{c})$ gene expressions in the retina of $C l k^{J r k}$ (a) and $c y c^{0}(\mathbf{b}, \mathbf{c})$ mutants. Data are normalized to the control $(C T R)$ as value 1 . Mann-Whitney test: $p<0.05$. Bars represent average of mRNA level $\pm \mathrm{SD}$ a
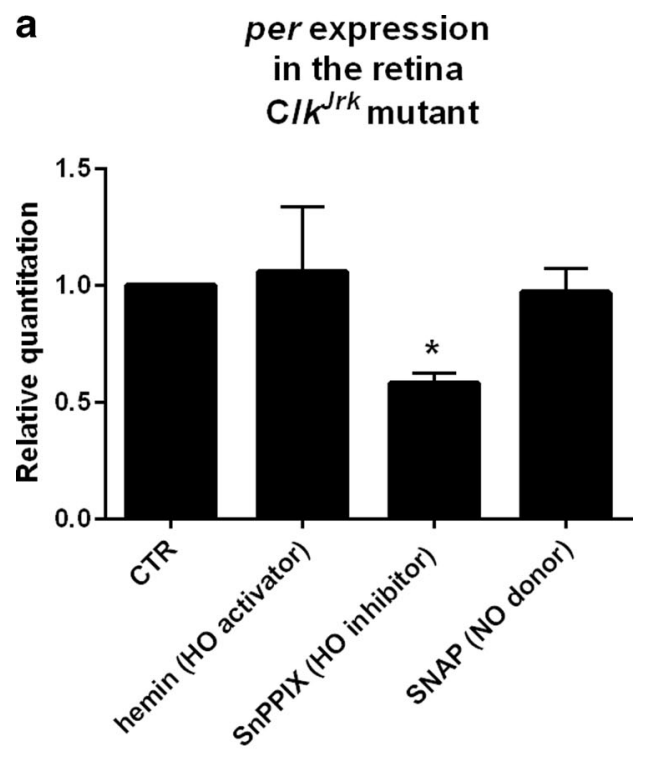

b
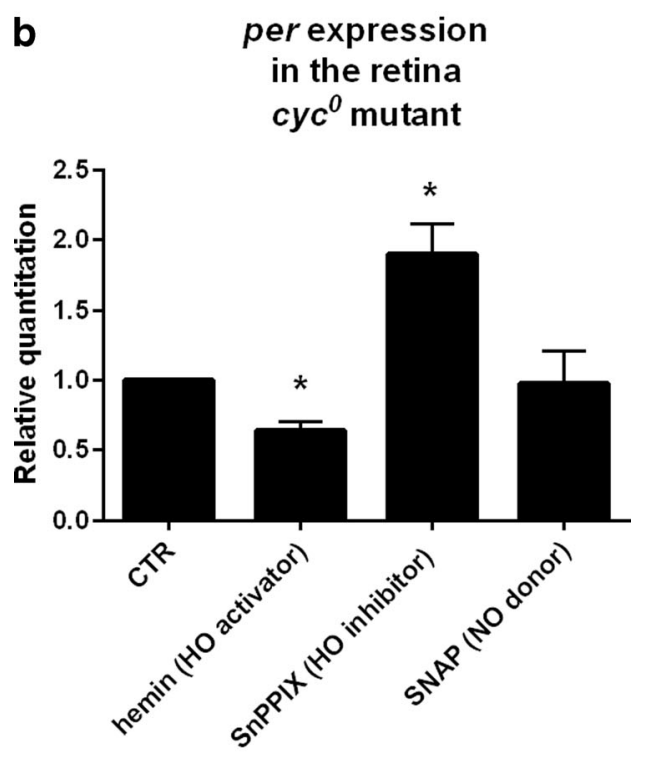

C

Clk expression

in the retina

cyc $^{0}$ mutant

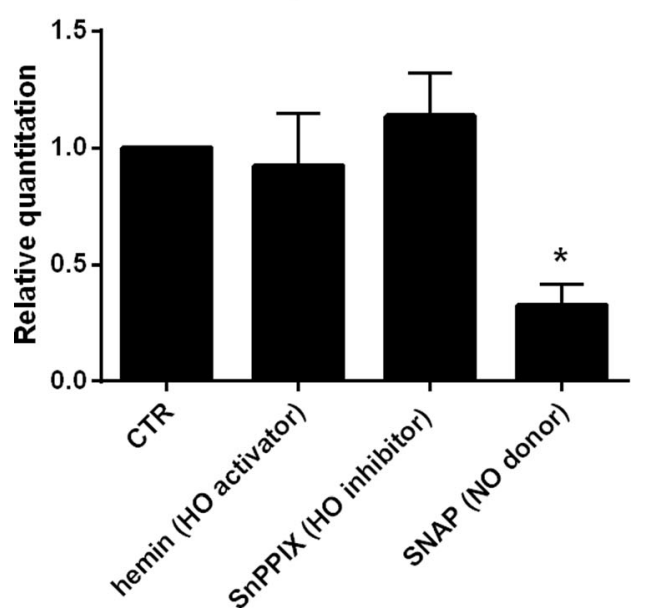




\section{Effect of HO, NO, and CO on Clock Gene Expression in $\mathbf{S 2}$ Cells}

In $\mathrm{S} 2$ cells cultured in the medium supplemented with $100 \mu \mathrm{M}$ of hemin, ho expression was increased (Fig. S7A). In the case of clock genes, per expression was decreased and $C l k$ increased after $6 \mathrm{~h}$ treatment with $100 \mu \mathrm{M}$ of hemin (Fig. 6a, b); however, $50 \mu \mathrm{M}$ of hemin had no effect on both gene expression (data not shown). In turn, $100 \mu \mathrm{M}$ of SnPPIX increased per gene expression and decreased $C l k$ expression after $6 \mathrm{~h}$ exposure to this HO inhibitor (Fig. 6a, b). NO donor, $100 \mu \mathrm{M}$ of SNAP, decreased per and increased Clk mRNA levels (Fig. 6c, d), however did not affect ho expression (Fig. S7B). The effect of CORM-2 was observed after $6 \mathrm{~h}$ exposure to $100 \mu \mathrm{M}$ concentration of CORM-2 (Fig. 6c, d). BV had no effect on per and Clk expressions (Fig. 6c, d).

\section{Effects of UV and White Light on DNA Damage in Photoreceptors}

When flies were fed with glucose and were not exposed to UV and white light, there were almost no changes in DNA integrity (Fig. 7c), while after the exposure the HRP signal was strong in the photoreceptor nuclei (Fig. 7d). This effect was even stronger after feeding flies with the HO inhibitor (Fig. 7e). However, flies fed with the HO activator and exposed to light had less DNA damage (Fig. 7f) than the control group fed with glucose. Their level of DNA damage in photoreceptors was similar to flies which were not exposed to UV and white light (Fig. 7c).

\section{Discussion}

\section{ho is Cyclically Expressed in the Retina and Protects Photoreceptors Against Degeneration}

We showed that in the retina of Drosophila, the ho gene, encoding heme oxygenase, oscillates in expression during the day, with two peaks at the beginning of the day (ZT1) and in the middle of the night (ZT16). This rhythm is maintained in constant darkness and is abolished in the arrhythmic per $^{01}$ null mutant. This means that ho is a clock-controlled gene (ccg). The expression of ho is probably controlled by the autonomous clock in the retina $[10,28,29]$ composed of circadian oscillators located in the retina photoreceptors. Their molecular mechanism is similar to that in the small $\mathrm{LN}_{\mathrm{v}} \mathrm{s}$, the main pacemaker neurons in the brain of Drosophila that are crucial for the circadian rhythm in locomotor activity [30,31]. However, as we showed in our previous study [10], rhythmic expression of some ccgs in the retina is also controlled by the pacemaker via the release of its two neurotransmitters, pigment-dispersing factor (PDF) and ion transport peptide. The cyclic expression of ho is also maintained in the brain since in whole head homogenates of $y w$ wild-type flies, the mRNA of ho peaks at ZT8 and ZT20 [9].
Fig. 6 Effects of hemin, SnPPIX, CORM-2, biliverdin, and SNAP treatments on per $(\mathbf{a}, \mathbf{c})$ and $C l k$ (b, d) gene expressions in $\mathrm{S} 2$ cells in vitro culture. RNA was isolated after $6 \mathrm{~h}$ of cell culturing with chemicals or control. Data are normalized to the control (CTR) as value 1 . Mann-Whitney test: $p<0.05$. Bars represent average clock gene expression level $\pm \mathrm{SD}$
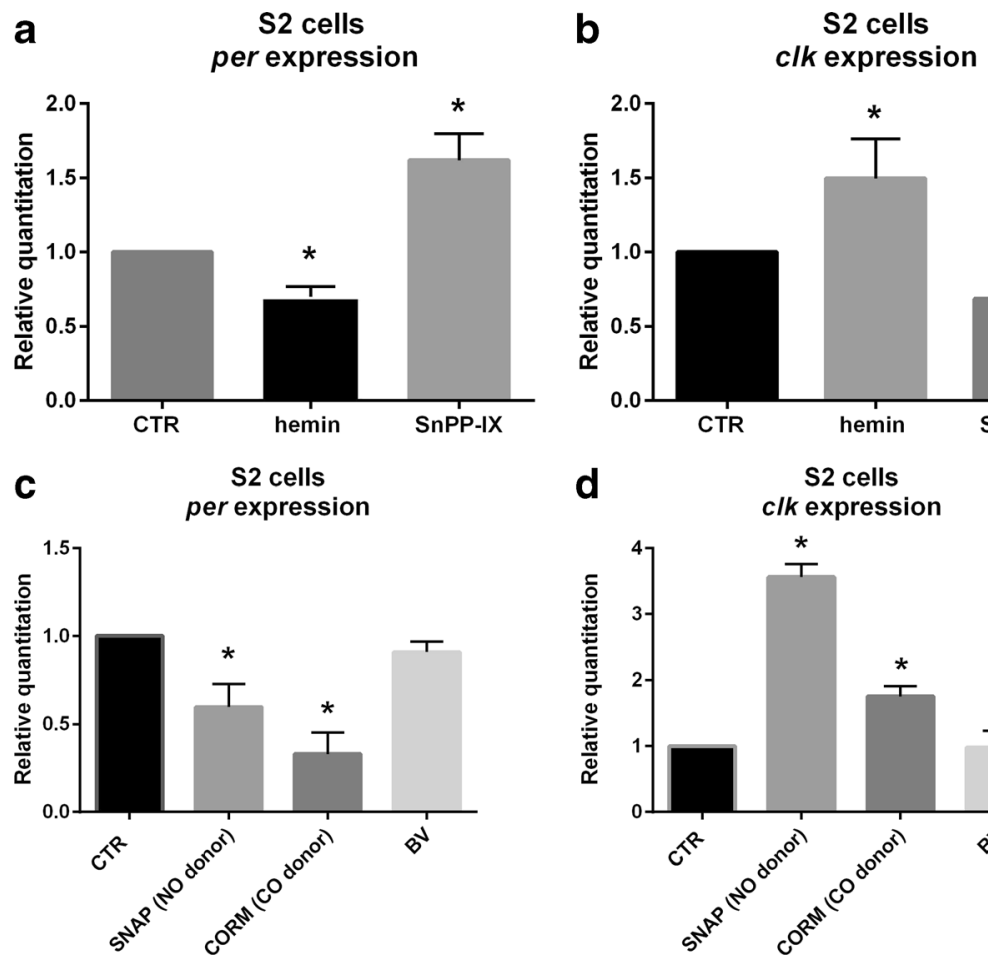

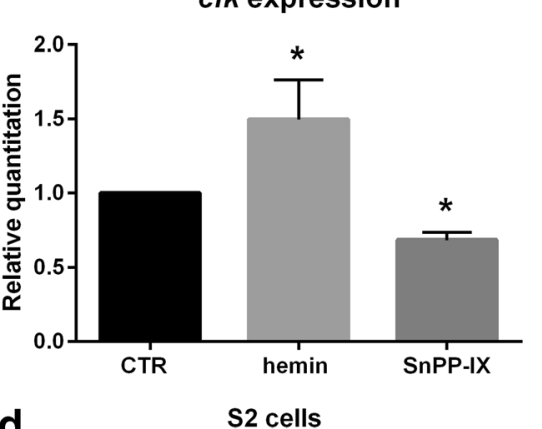

d $\begin{gathered}\text { S2 cells } \\ \text { d expression }\end{gathered}$

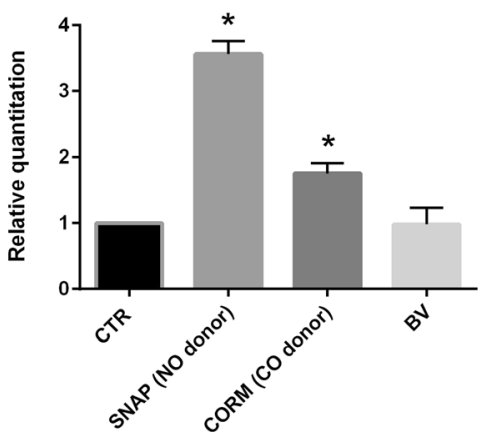



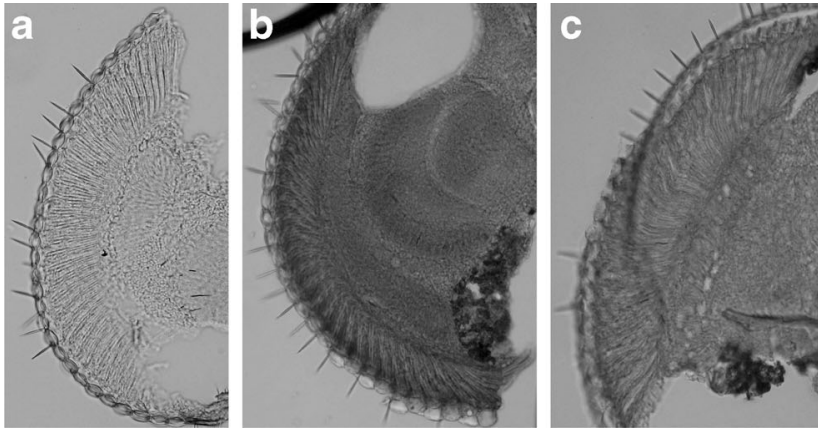

Fig. 7 Oxidative DNA damage after UV and intense white light exposure. DNA damage is visualized as dark color using anti-8hydroxyguanosine and DAB/HRP enzymatic reaction. a Negative control (without primary antibodies). b Positive control (heads incubated with

In mammals, as has been found in the SCN of hamsters, heme oxygenase is more active during the night (ZT16) than during the day (ZT4) in LD12:12; the same pattern is maintained in DD [5]. We suppose that in the retina of Drosophila, the high expression of $h o$ at the beginning of the day might be important for light adaptation of the photoreceptors and/or their protection during night/day transition. It has been reported that in mammals, ho expression is induced by ROS as well as by light pulse [32,33]. Since phototransduction increases the level of free radicals, this may cause cell damage and retina degeneration [15]. In addition to its role during the night/day transition, $\mathrm{HO}$ at ZT1 may also stimulate $C l k$ expression that is the highest at ZT4, affecting the clock. The second peak in ho expression, at ZT16, may be connected with the regulation of both per and Clk expressions since the activation of $\mathrm{HO}$ by hemin at ZT16 strongly suppresses per expression, while $C l k$ mRNA level is almost doubled than at the other time points during the day. The expression of ho is also cyclic in glial cells, with a peak at ZT16, so HO seems to participate in the regulation of cyclic processes in various cell types.

In the case of the retina photoreceptors, we found that $\mathrm{HO}$ protects them against ROS-induced degeneration, and when the HO level is low and photoreceptors are exposed to UV light, the DNA is seriously damaged.

\section{HO Influences the Molecular Clock}

It has already been reported in mammals that heme metabolism may modulate the circadian clock since it can synchronize clock gene expression in vitro [6]. In addition, heme injections increase $m P e r 1$ and decrease $m P e r 2$ clock gene expression in the mouse liver [6]. Heme can also bind to PER2 protein and induce its degradation [7]. This effect is tissue-specific since heme damps PER2 rhythm in the SCN, but not in other tissues as the thymus, esophagus, or spleen [8]. Moreover, oxidative stress reduces per cycling, with a weak effect in clock neurons and strong in peripheral
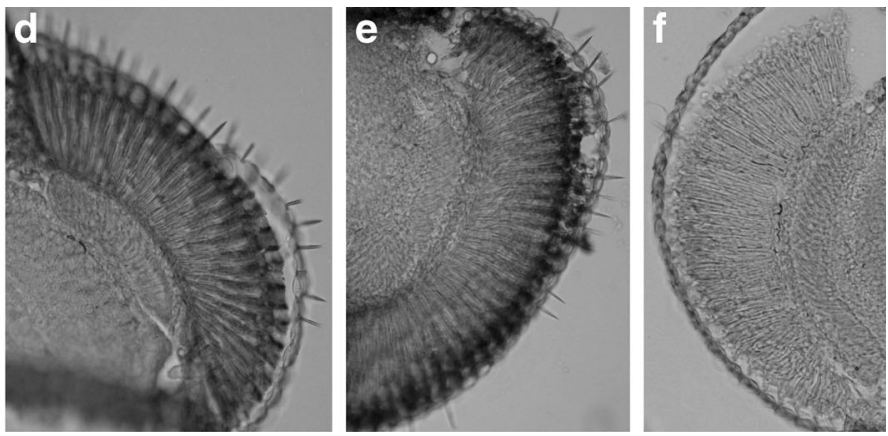

cytotoxic etoposide). c Control flies fed with glucose, not exposed to light. d Flies fed with glucose, exposed to light. e Flies fed with HO inhibitor (SNPPIX) and exposed to light. f Flies fed with HO activator (hemin) and exposed to light

oscillators, and decreases PDF level in the projections from $\mathrm{LN}_{\mathrm{v}} \mathrm{s}$ [34].

We found that the retina and glial peripheral clocks of Drosophila are also HO-dependent since transcription of clock main genes is affected by feeding flies with the heme oxygenase activator (hemin) or inhibitor (SnPPIX). In the pacemaker neurons of Drosophila, per and Clk genes are expressed in anti-phase and regulate each other's transcription (for a review, see [13]). In the retina, per and Clk mRNAs peak at ZT16 and ZT4, respectively. Hemin, which increases ho expression and $\mathrm{HO}$ activity, had dose-dependent effects on the expressions of per and Clk. It decreased per mRNA at each time point studied, except at ZT1, and increased $C l k$ mRNA, except at ZT4 and ZT13.

Moreover, the effects of chemical and genetic inhibition of ho expression were opposite on both genes' transcriptions, increasing per and decreasing $C l k$ mRNA levels. The opposite effects of the activator and inhibitor on per and Clk transcriptions at specific times during the day are not dependent on light, which could, for example, degrade those chemicals in food, because we obtained the same results in flies kept in DD (data not shown). This phenomenon probably results from changes of endogenous HO during the day. The high level of Clk mRNA at ZT1 after both hemin and SnPPIX is followed by the low level of per mRNA after applying both chemicals at ZT4. Moreover, per expression after hemin treatment was still rhythmic, with the same pattern of the rhythm, but with low amplitude. This indicates that HO damps per mRNA rhythm. In the case of $C l k$, the daily pattern of its expression was changed after feeding flies with hemin since the peak at ZT4 was abolished. It suggests that $\mathrm{HO}$ damps also Clk mRNA rhythm, but only at ZT4, while per mRNA is suppressed at the end of the night.

The obtained results indicate that $\mathrm{HO}$ regulates the molecular clock in the retina and in glial cells. Heme oxygenase decreases per expression and regulates the amplitude of $\mathrm{Clk}$ oscillations. The peak of $h o$ mRNA level is correlated with the maximum of per mRNA and seems to be crucial for 
regulating the clock mechanism. The high HO level at ZT16 inhibits per expression and decreases PER protein level after a few hours, at the end of the night. In the case of glial cells, the lack of HO abolishes per and Clk cyclic expressions, so the clock does not function in those cells.

The mechanism of heme oxygenase action on the molecular clock may be direct by binding to clock protein or transcription factors or indirectly by products of its activity $-\mathrm{CO}$, iron ions, or biliverdin. One might suppose that heme oxygenase influences the clock by regulating nitric oxide synthase activity or NO has an independent effect on the clock, as in the case of mammals [27].

The activity of clock proteins in mammals is regulated by binding $\mathrm{CO}$ or NO. It has been reported that the transcription factor NPAS2 can bind CO [35], while CLOCK can bind CO and NO [27]. Our results obtained using NO donor are similar to those after feeding flies with hemin since per mRNA level was decreased and $C l k$ expression was increased. In turn, feeding flies with CORM-2 did not show that $\mathrm{CO}$ is involved in the clock regulation. However, in S2 cells, we observed the inhibitory effect of CORM-2 on per and the stimulatory effect on $C l k$ expression. It may indicate that, in our experimental settings, feeding with CORM-2 was not sufficient to obtain the adequate concentration able to influence the clock mechanism in the retina. Moreover, $\mathrm{CO}$ action might be short-term. The effect of CORM-2 application was observed after $6 \mathrm{~h}$, but not after $12 \mathrm{~h}$ in S2 cell culture. We did not observe any impact of biliverdin, another $\mathrm{HO}$ activity product, on per and Clk transcriptions.

Using arrhythmic clock mutants $C l k^{\text {rrk }}$ and $c y c^{0}$, which do not synthesize CLK and CYC transcription factors, respectively, we found that in $C l k^{J r k}$ mutant fed with hemin or SNAP, there was no change of per expression, while SnPPIX treatment decreased per mRNA level. It means that CLK is involved in $\mathrm{HO}$ effect on per expression; however, $\mathrm{HO}$ may also activate other transcription factors which regulate the expression of per, and this process is NO-independent. In turn, in $c y c^{0}$ mutant, the effects of hemin or SnPPIX treatment on per expression were similar to those observed in wild-type flies; however, no effect was found after SNAP feeding. This suggests that CLK, but not CYC, is needed for the HO-dependent process of regulating per expression, but it does not involve NO.

\section{Conclusions}

HO seems to be a sensor of cell metabolism, and changes in $\mathrm{HO}$ activity affect the molecular mechanism of the circadian clock. This protein is important for cell survival, protecting cells against ROS-induced DNA damage and may suppress the clock under stressful conditions. HO increases the expression of $\mathrm{Clk}$, and this process seems to depend on the $\mathrm{CO}$ and
NO levels. NO increases $C l k$ expression, but may also decrease it when the CYC protein is missing. In turn, the expression of per is decreased after the activation of HO. The expression of per may be additionally regulated by $\mathrm{HO}$ in a CLK-independent pathway, or per may have in the Drosophila visual system another function regulated by HO. HO seems to regulate the clock at the beginning of the day and at the end of the night by different processes.

Acknowledgments This study was supported by the National Science Centre in Poland grant no. 2012/07/B/NZ3/02908 to EP. The Faculty of Biochemistry, Biophysics and Biotechnology of the Jagiellonian University is a beneficiary of the structural funds from the European Union and the Polish Ministry of Science and Higher Education (grant nos. POIG.02.01.00-12-064/ 08 and 02.02.00-00-014/08) and is a partner of the Leading National Research Center (KNOW) supported by the Ministry of Science and Higher Education. We would like to thank Dr. Charalambos P. Kyriacou (University of Leicester, Leicester, UK) and Dr. Amita Sehgal (University of Pennsylvania Perelman School of Medicine, Philadelphia, USA) for reading the manuscript and for their useful comments.

Authors' Contributions MD and AL have carried out experiments and analyzed the data. EP wrote the manuscript. JD and AJ donated chemicals, helped with methods, and discussed results and the final version of the manuscript.

Open Access This article is distributed under the terms of the Creative Commons Attribution 4.0 International License (http:// creativecommons.org/licenses/by/4.0/), which permits unrestricted use, distribution, and reproduction in any medium, provided you give appropriate credit to the original author(s) and the source, provide a link to the Creative Commons license, and indicate if changes were made.

\section{References}

1. Loboda A, Jazwa A, Grochot-Przeczek A, Rutkowski AJ, Cisowski J, Agarwal A, Jozkowicz A, Dulak J (2008) Heme oxygenase-1 and the vascular bed: from molecular mechanisms to therapeutic opportunities. Antioxid Redox Signal 10:1767-1812

2. Zhang X, Sato M, Sasahara M, Migita CT, Yoshida T (2004) Unique features of recombinant heme oxygenase of Drosophila melanogaster compared with those of other heme oxygenases studied. Eur J Biochem 271:1713-1724

3. Cui L, Yoshioka Y, Suyari O, Kohno Y, Zhang X, Adachi Y, Ikehara S, Yoshida T et al (2008) Relevant expression of Drosophila heme oxygenase is necessary for the normal development of insect tissues. Biochem Biophys Res Commun 377:1156-1161

4. Ida H, Suyari O, Shimamura M, Tien Tai T, Yamaguchi M, Taketani S (2013) Genetic link between heme oxygenase and the signaling pathway of DNA damage in Drosophila melanogaster. Tohoku J Exp Med 231:117-125

5. Rubio MF, Agostino PV, Ferreyra GA, Golombek DA (2003) Circadian heme oxygenase activity in the hamster suprachiasmatic nuclei. Neurosci Lett 353:9-12

6. Kaasik K, Lee CC (2004) Reciprocal regulation of haem biosynthesis and the circadian clock in mammals. Nature 430:467-471

7. Yang J, Kim KD, Lucas A, Drahos KE, Santos CS, Mury SP, Capelluto DG, Finkielstein CV (2008) A novel heme-regulatory motif mediates heme-dependent degradation of the circadian factor period 2. Mol Cell Biol 28:4697-4711 
8. Guenthner CJ, Bickar D, Harrington ME (2009) Heme reversibly damps PERIOD2 rhythms in mouse suprachiasmatic nucleus explants. Neuroscience 164:832-841

9. Ceriani MF, Hogenesch JB, Yanovsky M, Panda S, Straume M, Kay SA (2002) Genome-wide expression analysis in Drosophila reveals genes controlling circadian behavior. J Neurosci 22:93059319

10. Damulewicz M, Loboda A, Bukowska-Strakowa K, Jozkowicz A, Dulak J, Pyza E (2015) Clock and clock-controlled genes are differently expressed in the retina, lamina and in selected cells of the visual system of Drosophila melanogaster. Front Cell Neurosci 9: 353

11. Damulewicz M, Rosato E, Pyza E (2013) Circadian regulation of the $\mathrm{Na}^{+} / \mathrm{K}^{+}$-ATPase alpha subunit in the visual system is mediated by the pacemaker and by retina photoreceptors in Drosophila melanogaster. PLoS One 8, e73690

12. Górska-Andrzejak J, Makuch R, Stefan J, Görlich A, Semik D, Pyza E (2013) Circadian expression of the presynaptic active zone protein Bruchpilot in the lamina of Drosophila melanogaster. Dev Neurobiol 73:14-26

13. Ozkaya O, Rosato E (2012) The circadian clock of the fly: a neurogenetics journey through time. Adv Genet 77:79-123

14. Collins B, Mazzoni EO, Stanewsky R, Blau J (2006) Drosophila CRYPTOCHROME is a circadian transcriptional repressor. Curr Biol 16:441-449

15. Chartier FJ, Hardy ÉJ, Laprise P (2012) Crumbs limits oxidasedependent signaling to maintain epithelial integrity and prevent photoreceptor cell death. J Cell Biol 198:991-998

16. Pyza E (2010) Circadian rhythms in the fly's visual system. In: Dartt DA (ed) Encyclopedia of the eye. Academic, Oxford, pp 302-311

17. Allada R, White NE, So WV, Hall JC, Rosbash M (1998) A mutant Drosophila homolog of mammalian Clock disrupts circadian rhythms and transcription of period and timeless. Cell 93:791-804

18. Rutila JE, Suri V, Le M, So WV, Rosbash M, Hall JC (1998) CYCLE is a second bHLH-PAS clock protein essential for circadian rhythmicity and transcription of Drosophila period and timeless. Cell 93:805-814

19. Sehgal A, Price JL, Man B, Young MW (1994) Loss of circadian behavioral rhythms and per RNA oscillations in the Drosophila mutant timeless. Science 263:1603-1606

20. Mollereau B, Wernet MF, Beaufils P, Killian D, Pichaud F, Kühnlein R, Desplan C (2000) A green fluorescent protein enhancer trap screen in Drosophila photoreceptor cells. Mech Dev 93: $151-160$

21. Ni JQ, Liu LP, Binari R, Hardy R, Shim HS, Cavallaro A, Booker M, Pfeiffer BD et al (2009) A Drosophila resource of transgenic RNAi lines for neurogenetics. Genetics 182:1089-1100
22. Schneider I (1972) Cell lines derived from late embryonic stages of Drosophila melanogaster. J Embryol Exp Morphol 27:353-365

23. Ilmarinen-Salo P, Moilanen E, Kinnula VL, Kankaanranta H (2012) Nitric oxide-induced eosinophil apoptosis is dependent on mitochondrial permeability transition (mPT), JNK and oxidative stress: apoptosis is preceded but not mediated by early mPT-dependent JNK activation. Respir Res 13:73

24. Tataroglu O, Emery P (2014) Studying circadian rhythms in Drosophila melanogaster. Methods 68:140-150

25. Durante W, Kroll MH, Christodoulides N, Peyton KJ, Schafer AI (1997) Nitric oxide induces heme oxygenase-1 gene expression and carbon monoxide production in vascular smooth muscle cells. Circ Res 80:557-564

26. Hartsfield CL, Alam J, Cook JL, Choi AM (1997) Regulation of heme oxygenase-1 gene expression in vascular smooth muscle cells by nitric oxide. Am J Physiol 273:L980-988

27. Lukat-Rodgers GS, Correia C, Botuyan MV, Mer G, Rodgers KR (2010) Heme-based sensing by the mammalian circadian protein CLOCK. Inorg Chem 49:6349-6365

28. Siwicki KK, Eastman C, Petersen G, Rosbash M, Hall JC (1988) Antibodies to the period gene product of Drosophila reveal diverse tissue distribution and rhythmic changes in the visual system. Neuron 1:141-150

29. Zerr DM, Hall JC, Rosbash M, Siwicki KK (1990) Circadian fluctuations of period protein immunoreactivity in the CNS and the visual system of Drosophila. J Neurosci 10:2749-2762

30. Blanchardon E, Grima B, Klarsfeld A, Chélot E, Hardin PE, Préat T, Rouyer F (2001) Defining the role of Drosophila lateral neurons in the control of circadian rhythms in motor activity and eclosion by targeted genetic ablation and PERIOD protein overexpression. Eur J Neurosci 13:871-888

31. Helfrich-Förster C (1998) Robust circadian rhythmicity of Drosophila melanogaster requires the presence of lateral neurons: a brain-behavioral study of disconnected mutants. J Comp Physiol A $182: 435-453$

32. Ben-Shlomo R, Akhtar RA, Collins BH, Judah DJ, Davies R, Kyriacou CP (2005) Light pulse-induced heme and ironassociated transcripts in mouse brain: a microarray analysis. Chronobiol Int 22:455-471

33. Panchenko MV, Farber HW, Korn JH (2000) Induction of heme oxygenase-1 by hypoxia and free radicals in human dermal fibroblasts. Am J Physiol Cell Physiol 278:C92-C101

34. Zheng X, Yang Z, Yue Z, Alvarez JD, Sehgal A (2007) FOXO and insulin signaling regulate sensitivity of the circadian clock to oxidative stress. Proc Natl Acad Sci U S A 104:15899-15904

35. Dioum EM, Rutter J, Tuckerman JR, Gonzalez G, Gilles-Gonzalez MA, McKnight SL (2002) NPAS2: a gas-responsive transcription factor. Science 298:2385-2387 